\title{
Catastrophic antiphospholipid syndrome - a case report of a highly fatal disease
}

\author{
Authors: Nishita Padmanabahan ${ }^{A}$ and Sanjeev Jalihal ${ }^{B}$
}

\section{Aim}

The aim was to highlight the importance of recognising a highly fatal disease, catastrophic antiphospholipid syndrome (CAPS).

\section{Summary}

The following is a case report of a 58-year-old man that presented with left leg cramp after returning from a holiday. While the Doppler ultrasound showed no evidence of deep-vein thrombosis, he presented a few days later with chest pain and shortness of breath. Computed tomography (CT) pulmonary angiography showed that he had bilateral pulmonary emboli and also evidence of mediastinal and cervical lymphadenopathy. The scan also raised the possibility of lymphoma. He was commenced on anticoagulation and referred to the haematologist who arranged a CT of the neck, chest, abdomen and pelvis which confirmed multiple bilateral lung nodules with mediastinal and low-volume neck lymphadenopathy. He was then referred to the respiratory physicians who suggested a biopsy.

Within a few days, the patient developed left arm weakness, left-sided facial droop and slurred speech. A CT of the head showed incidental aneurysm, and further imaging with magnetic resonance imaging revealed likely brain metastasis. The patient was commenced on steroids and went on to have the lung biopsy, which later confirmed adenocarcinoma of the lung. The patient presented similarly and was diagnosed with multiple cerebral and cerebellar infarcts as well as infarcts in the kidney and spleen.

At his next presentation, the patient was septic in type 2 respiratory failure with new parenchymal infarcts in the kidneys and spleen with a high suspicion of a bowel infarct. Fever, thrombocytopenia, neurological abnormalities, acute kidney injury and the presence of schistocytes in the blood film with an elevated lactate dehydrogenase suggested a diagnosis of thrombotic thrombocytopenic purpura. The patient underwent plasmapheresis and blood results showed normal ADAMTS13 activity with positive anticardiolipin antibody and anti-beta-2 glycoprotein I antibody.

Authors: ${ }^{A}$ internal medicine trainee, Scunthorpe General Hospital, Scunthorpe, UK; ${ }^{B}$ consultant haematologist, Scunthorpe General Hospital, Scunthorpe, UK

\section{Results}

A diagnosis of CAPS was made, and the patient eventually succumbed despite anticoagulation and plasmapheresis.

\section{Conclusions}

We hope that this case report raises awareness among physicians and helps ensure early diagnosis and prompt treatment of CAPS.

\section{Conflicts of interest}

None declared. 